\title{
Aplicación de videotutoriales para la mejora de las competencias matemáticas en los estudiantes de pregrado de la Universidad de San Martín de Porres
}

\section{Application of video tutorials for the improvement of mathematical competencies in undergraduate students at Universidad de San Martín de Porres.}

DOI: $10.46932 / \mathrm{sfjdv2n2-002}$

Received in: January 1st, 2020

Accepted in: March 31th, 2020

\section{Mg. José Dolores Esquerre Quispe}

Doctorado en Educación de la Universidad de San Martín de Porres. Maestría en Educación con mención en Informática y Tecnología Educativa. Licenciado en Matemática, Física, Estadística e Informática y Nuevas Tecnologías en la UNED Perú. Especializaciones en Andragogía, Gestión Educativa y Docencia Virtual. Especialista en Educación Virtual en el Ministerio de Educación.

Universidad de San Martín de Porres - Lima 013, Lima, Perú

Correos: jesquerre@usmpvirtual.edu.pe

\section{Dra. Milagros Cecilia Huamán Castro}

Doctora en Educación graduada en la Universidad de San Martín de Porres - Perú. Posdoctoral PhD

“Digital Learning y Experiencias de Aprendizaje Emergente" Universidad de Barcelona - España.

Magister en Docencia e Investigación Universitaria. Licenciada en Lengua y Literatura, graduada en la Facultad de Educación de la Universidad de San Martín de Porres - USMP. Especialización Profesional en

Andragogía para Jóvenes y Adultos. II Especialización Profesional en Gerencia y Gestión Educativa.

Especialización en Docencia Virtual. Especialización de Posgrado en Gestión Universitaria.

Universidad de San Martín de Porres - Lima 013, Lima, Perú

Correos: mhuaman@usmpvirtual.edu.pe

\section{RESUMEN}

La investigación tuvo como objetivo principal determinar de qué manera influye el uso de video tutoriales en la mejora de las competencias educativas de los estudiantes de Matemática de pregrado en las aulas virtuales de la USMP el año 2018. El tipo de investigación fue con enfoque tecnológico y de corte transversal, cuyo método de investigación fue cuasi-experimental. La población estuvo formada por los estudiantes de pregrado de la asignatura de Matemática I 2018 de la USMP Virtual (USMP) quienes llevaron la asignatura en el aula virtual. Los estudiantes fueron un total de 60 integrantes. Ellos fueron matriculados según estricto orden de llegada, hasta acabarse las vacantes. El instrumento que se utilizó en la investigación relacionada a las competencias matemáticas, fue una rúbrica que cuenta con un total de 15 preguntas. Los resultados obtenidos permitieron concluir que el uso del video tutoriales ayuda a la mejora de las competencias matemáticas de los estudiantes de Matemática de pregrado en las aulas virtuales de la USMP Virtual 2018.

Palabras clave: Matemática, videotutoriales, competencias, e-learning, conectivismo.

\section{ABSTRACT}

The main objective of the research was to determine how the use of video tutorials influences the improvement of educational skills of undergraduate Mathematics students in the virtual classrooms of the USMP in 2018. The type of research was with technological approach and cross-sectional, whose research method was quasi-experimental. The population was formed by the undergraduate students of the subject of Mathematics I 2018 of the USMP Virtual (USMP) who took the subject in the virtual classroom. The students were a total of 60 members. They were enrolled according to strict order of arrival, until the vacancies ran out. The instrument used in the research related to mathematical competencies was a rubric 
with a total of 15 questions. The results obtained allowed us to conclude that the use of video tutorials helps to improve the mathematical competencies of undergraduate Mathematics students in the virtual classrooms of the USMP Virtual 2018.

Keywords: Mathematics, video tutorials, competencies, e-learning, connectivism.

\section{INTRODUCCIÓN}

Los estudiantes de pregrado semipresencial inician la asignatura de Matemática con conocimientos básicos. En algunos casos, estos son muy escasos y aún más, si son estudiantes adultos es más difícil recuperar esos conocimientos. Adicional a ello el otro problema que se afronta es el poco uso de la tecnología, algunos de ellos es la primera vez que utilizan un aula virtual. Es la primera vez que comparten una comunicación con un tutor virtual a través de foros, chat o con mensajes a través de los correos internos y videoconferencias, lo cual se suma a la complejidad que una asignatura de números tiene por naturaleza. Los estudiantes participan en las asignaturas de Matemática I y Matemática II a través de foros temáticos, que evidencian su participación para ser evaluados; también desarrollan tareas que consisten en resolución de problemas seleccionados por el tutor, pero para ello, previamente revisan los materiales que tienen en el aula virtual, como los videos tutoriales. Estos videos tutoriales por lo general son seleccionados de la web desde YouTube. Se cuenta con un foro temático donde desarrollan sus ejercicios y los comparten con sus compañeros, quienes debaten sobre la forma cómo resolver estos ejercicios y/o problemas. En este proceso se puede observar que no todos realizan las consultas, muchos se quedan en silencio virtual. Muchos de los estudiantes cuando inician esta asignatura, se retiran pronto, y si continúan, culminan con bajo rendimiento académico; hay poca predisposición a las matemáticas. Cuentan con sesiones de videoconferencia, que les permiten resolver problemas o ejercicios similares, pero ingresan muy pocos estudiantes. La web nos ofrece un abanico de herramientas gratuitas que están en proceso de investigación, para experimentar su uso y aplicación, en pro de una mejora continua de la calidad educativa en nuestros estudiantes, pero muy pocos tutores las investigan y aplican, la gran mayoría por desconocimiento del manejo de la tecnología, y otros por la resistencia al cambio. Ante ello, se decidió aplicar el uso de videotutoriales creados por el mismo tutor para cada sesión de videoconferencia, de manera muy personalizada y didáctica, lo cual comenzó a motivar y despertar interés en ellos. Además, al revisarlos las veces que ellos necesitaban libremente, les permitió mejorar sus competencias. Por ello, se comenzó a aplicar los videotutoriales como parte del material obligatorio por parte del docente, quien requiere contar con capacidades tecnológicas frente a las necesidades de los estudiantes, y la USMP los capacita permanentemente en estas. 


\section{CONECTIVISMO}

Siemens (2004) en su "Teoría de aprendizaje para la era digital" indica que un principio central de la mayoría de las teorías del aprendizaje es que el aprendizaje ocurre dentro de una persona. Incluso los enfoques del constructivismo social, los cuales sostienen que el aprendizaje es un proceso social, promueven el protagonismo del individuo en el aprendizaje. Estas teorías no hacen referencia al aprendizaje que ocurre por fuera de las personas (el aprendizaje que es almacenado y manipulado por la tecnología). Siemens nos indica que, también fallan al describir cómo ocurre el aprendizaje al interior de las organizaciones.

Principios del Conectivismo:

- El aprendizaje y el conocimiento dependen de la diversidad de opiniones.

- El aprendizaje es un proceso de conectar nodos o fuentes de información especializados.

- El aprendizaje puede residir en dispositivos no humanos.

- La capacidad de saber más es más crítica que aquello que se sabe en un momento dado.

- La alimentación y mantenimiento de las conexiones es necesaria para facilitar el aprendizaje continuo.

- La habilidad de ver conexiones entre áreas, ideas y conceptos es una habilidad clave.

- La actualización (conocimiento preciso y actual) es la intención de todas las actividades conectivistas de aprendizaje.

El punto de partida del Conectivismo es el individuo. El conocimiento personal se compone de una red, la cual alimenta a organizaciones e instituciones, las que a su vez retroalimentan a la red, proveyendo nuevo aprendizaje para los individuos.

\section{METODOLOGÍAS DE EDUCACIÓN VIRTUAL}

Según Lara (2012). La metodología responde al cómo enseñar y aprender. Y en cada modelo de educación virtual se destaca la metodología como base del proceso. A continuación, él destaca tres métodos más sobresalientes: el método sincrónico, asincrónico y aula virtual - presencial.

Método sincrónico: Estos recursos sincrónicos se hacen verdaderamente necesarios como agente socializador, imprescindible para que el alumno que estudia en la modalidad a virtual no se sienta aislado. Son: videoconferencias con pizarra, audio o imágenes como el NetMeeting de Internet, Chat, Chat de voz, audio y asociación en grupos virtuales.

Método asincrónico: Son más valiosos para su utilización en la modalidad de educación a distancia, ya que el acceso en forma diferida en el tiempo de la información se hace absolutamente necesario por las características especiales que presentan los alumnos que estudian en esta modalidad virtual (limitación de tiempos, cuestiones familiares y laborales, etc.). Son Email, foros de discusión, 
www., textos, gráficos animados, audio, CDS interactivos, video, casetes etc.

Según Álvarez (2012) en la educación virtual interactúan cuatro variables: el maestro y el alumno; la tecnología y el medio ambiente. En cambio, Banet (2011) se adelanta en su concepción y afirma: "la educación virtual es una combinación entre la tecnología de la realidad virtual, redes de comunicación y seres humanos. En los próximos, la educación virtual será de extender y tocar a alguien o una población entera de una manera que los humanos nunca experimentaron anteriormente"

Huamán (2015) en sus publicaciones precisa que la metodología para sesiones de clase virtual se basa en los fundamentos del Constructivismo, en el cual el docente no es el centro del proceso de aprendizaje, ni tampoco lo es el contenido como en el Conductivismo. No existe el dictado ni trasferencia de conocimientos, sino el acompañamiento del tutor /docente, para lograr un aprendizaje significativo a lo largo del proceso. Tanto el docente como el alumno cumplen diferentes funciones en la educación virtual, pero, finalmente, tienen un punto de intersección, el aprendizaje significativo. Es así que es indispensable que el docente virtual conozca las herramientas tecnológicas de la Web 2.0 (Moodle LMS, Canvas LMS, Chamilo LMS; Redes sociales; Servicio Micro Blogging, etc.).

Huamán (2015) manifiesta que en la USMP Virtual se consideró trabajar como parte de la metodología virtual, la metacognición ya que esta implica reflexionar sobre nuestro propio pensamiento o conocimiento sobre nuestros aprendizajes, así como sobre nosotros mismos en cuanto a aprendices. Después de estudiar distintas definiciones y averiguaciones empíricas de la metacognición, concluye que es importante que los seres humanos se comprendan así mismos como agentes de su propio pensamiento nuestro pensamiento puede ser controlado y regulado de forma deliberada. Lo cual es muy importante en el desarrollo académico en el entorno virtual.

\section{VIDEOTUTORIALES}

Gonzales (2013) la multimedia surge como una herramienta poderosa de apoyo a la educación permitiendo al docente del diseño de sus tareas de manera diferente. La multimedia requiere de una planeación para su incorporación en la educación ya que su éxito no solo depende de que el material esté atractivo, si no de que los objetivos para los que se diseñó se trabajen de manera correcta.

Las estrategias de aprendizaje deben estar conformadas por la parte informativa, planear la actuación del alumno, monitorear la ejecución y por último evaluar los resultados obtenidos para que esta funcione como se espera. "El uso de multimedia para la elaboración de estrategias de aprendizaje" el docente hoy más que nunca es facilitador del aprendizaje y siempre debe preparar oportunidades de aprendizaje para sus alumnos, es fundamental que estimule el deseo de aprender de estos. Gonzales (2013). 


\section{EDUCADORES VIRTUALES}

Ser educador virtual también llamado tutor será una de las opciones más cotizadas en el siglo XXI. No todos los docentes están dispuestos a renunciar a sus clases magistrales, así que el educador virtual, además de desarrollar una de las profesiones con más futuro en la Nueva Economía, si está convertido en el ente más buscado por universidades y escuelas de negocios. Según Bricall (2002), Rector de la universidad de Barcelona, afirma: "la introducción de las nuevas tecnologías en la educación no supone la desaparición del profesor, aunque obliga a establecer un nuevo equilibrio en sus funciones". En cambio, Asencio (2002) sistematiza las características de un Educador virtual de la siguiente manera.

\section{METODOLOGÍA DE LA INVESTIGACIÓN}

El tipo de investigación fue con enfoque tecnológico y de corte transversal, cuyo método de investigación es cuasiexperimental porque se describieron dos variables y analizaron su interrelación en un momento único del tiempo. Además, se analizó directamente la relación de dos variables para conocer la mejora de las competencias educativas con el uso de los video tutoriales en un aula virtual de pregrado.

Se consideró un grupo experimental (GE) y un grupo control (GC) a los cuales Se les aplicó una evaluación pre test $(\mathrm{O} 1)$, luego se aplicó un tratamiento $(\mathrm{X})$ al grupo experimental y finalmente, se aplicó una evaluación postest (O2) a cada grupo:

Se desarrolló la primera unidad de trabajo en la resolución de ejercicios y problemas de matemática de forma tradicional en el aula virtual, enviando sus productos al tutor por el espacio indicado para las tareas y actividades.

Se aplicó el pre test a los dos grupos. El pre test evaluó las tres dimensiones de las competencias educativas (competencia conceptual, competencia procedimental y competencia actitudinal) de los estudiantes.

Se elaboró y aplicó videotutoriales creados por el docente sobre la resolución de ejercicios y problemas, tanto en las tareas y actividades, como en los foros temáticos y foros de consulta, para los estudiantes de los equipos de trabajo que forman parte del grupo experimental (GE).

Se desarrolló la segunda unidad. En esta unidad, los equipos que son parte del grupo control (GC) desarrollaron la segunda unidad en forma convencional, mientras que los equipos que son parte del grupo experimental (GE) lo desarrollaron utilizando los videotutoriales elaborados por el docente.

Se compararon los resultados de las mediciones obtenidas en las evaluaciones pre test y post test en el grupo experimental, de las evaluaciones pre test y post test en el grupo de control. Luego se procedió al análisis comparativo de los resultados de las evaluaciones postest del grupo experimental y del grupo de control.

Se verificó si las comparaciones de las mediciones realizadas permitieron alcanzar los objetivos y contrastar las hipótesis. 


\subsection{DISEÑO MUESTRAL}

La población estuvo formada por los estudiantes de pregrado de la asignatura de Matemática I 2018 de la USMP Virtual (USMP). Estos estudiantes llevaron la asignatura en el aula virtual. Los estudiantes fueron un total de 60 integrantes. Ellos fueron matriculados según estricto orden de llegada, hasta acabarse las vacantes.

El tipo de muestreo fue no probabilístico, de sujetos tipo, ya que la muestra fue determinada de manera directa, debido a la designación del dictado de este grupo en el periodo 2018, por ello aproveché esa oportunidad, ya que me hice cargo del grupo de estudiantes de esta asignatura del Pregrado de la USMP Virtual y estuvieron integrados oficialmente por un total de 60 estudiantes. Luego para efectos de la investigación se formaron dos grupos de acuerdo con el orden de matrícula, grupos de 30 estudiantes cada uno de acuerdo con la lista que emitió la Dirección de estudios.

Se determinaron los grupos de estudio:

Grupo experimental (GE): Estudiantes con quienes se desarrollaron las sesiones de clase en el aula virtual con apoyo de los videotutoriales, formado por 30 estudiantes de la asignatura de Matemática I del pregrado en la USMP Virtual el año 2018.

Grupo control (GC): Estudiantes con quienes se desarrollaron las sesiones de clase en el aula virtual de manera convencional, formado por 30 estudiantes de la asignatura de Matemática I del pregrado en la USMP Virtual el año 2018.

Ambos grupos conformaron un total de 60 estudiantes de aulas virtuales de pregrado, matriculados en la asignatura de Matemática I del pregrado de la USMP Virtual del año 2018.

\section{RESULTADOS}

Tabla 7

Descripción de los niveles de las competencias matemáticas

\begin{tabular}{lcccccccc}
\hline & \multicolumn{2}{l}{ GC Pretest } & GC Post test & GE Pretest & \multicolumn{2}{c}{ GE Post test } \\
& $\mathrm{f}$ & $\%$ & $\mathrm{f}$ & $\%$ & $\mathrm{f}$ & $\%$ & $\mathrm{f}$ & $\%$ \\
\hline Logrado & 0 & $0 \%$ & 0 & $0,0 \%$ & 0 & $0 \%$ & 30 & $100 \%$ \\
& & & & & & & & \\
\hline Proceso & 20 & $33 \%$ & 29 & $97 \%$ & 23 & $77 \%$ & 0 & $0 \%$ \\
\hline Inicio & 10 & $67 \%$ & 1 & $3 \%$ & 7 & $23 \%$ & 0 & $0 \%$ \\
\hline Total & 30 & $100,0 \%$ & 30 & $100,0 \%$ & 30 & $100,0 \%$ & 30 & $100,0 \%$ \\
\hline
\end{tabular}

Fuente. Base de datos 
Figura 1. Porcentajes de los niveles de la variable competencias matemáticas

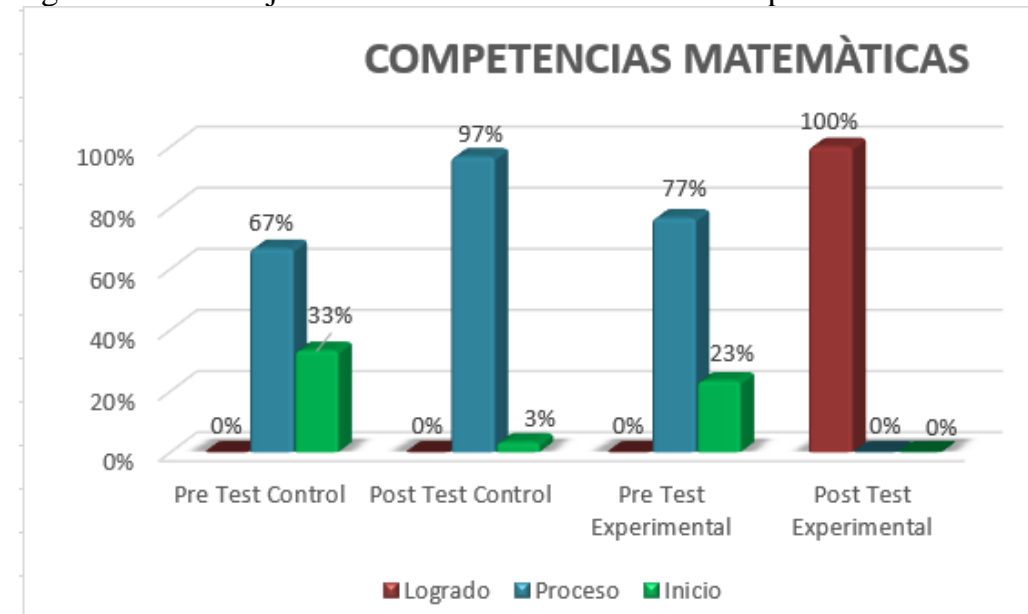

Los datos reflejados al haber realizado tabla y figura respectiva, en cuanto a la evaluación realizada antes de haber aplicado la intervención, muestran respecto al grupo control que el $33 \%$ de los estudiantes estaban en el nivel de inicio, mientras que el 67\% llegó al nivel de proceso; en el grupo experimental se evidenció que existía un $23 \%$ que se encontraban en el nivel de inicio y el $77 \%$ en el nivel proceso.

Después de realizada la intervención se volvió a evaluar encontrándose que en el grupo control el 3\% se ubicó en el nivel de inicio y el 97\% llegó al nivel proceso, mientras que en el grupo experimental el 100\% logró ubicarse en el nivel logrado en cuanto a la competencia matemática.

Figura 2. Porcentajes de los niveles de la competencia conceptual

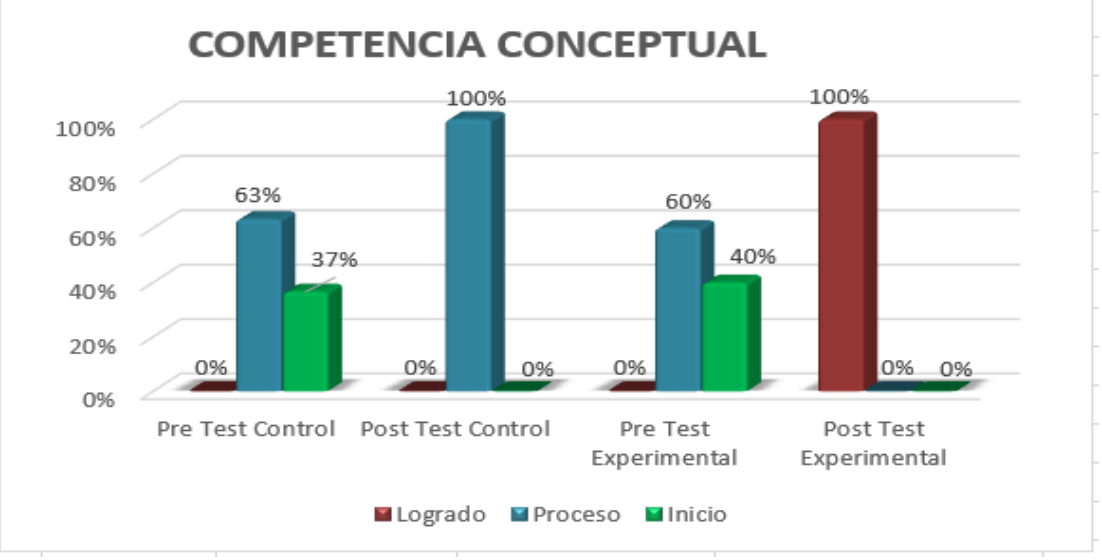

En cuanto a la competencia conceptual, los datos presentados en la tabla y figura muestran que la evaluación realizada antes de la intervención en el grupo-control fue que el 37\% se ubicaron en el nivel de inicio, el $63 \%$ en el nivel de proceso, mientras que en el grupo experimental se tuvo que el $40 \%$ estaba en el nivel de inicio y el $60 \%$ en el nivel de proceso.

Después de realizada la intervención se procedió a evaluar a los estudiantes obteniéndose que en el grupo control logró el 93\% del nivel de proceso, y el grupo experimental logró el 100\% 
Figura 3. Porcentajes de los niveles de la competencia procedimental

\section{COMPETENCIA PROCEDIMENTAL}

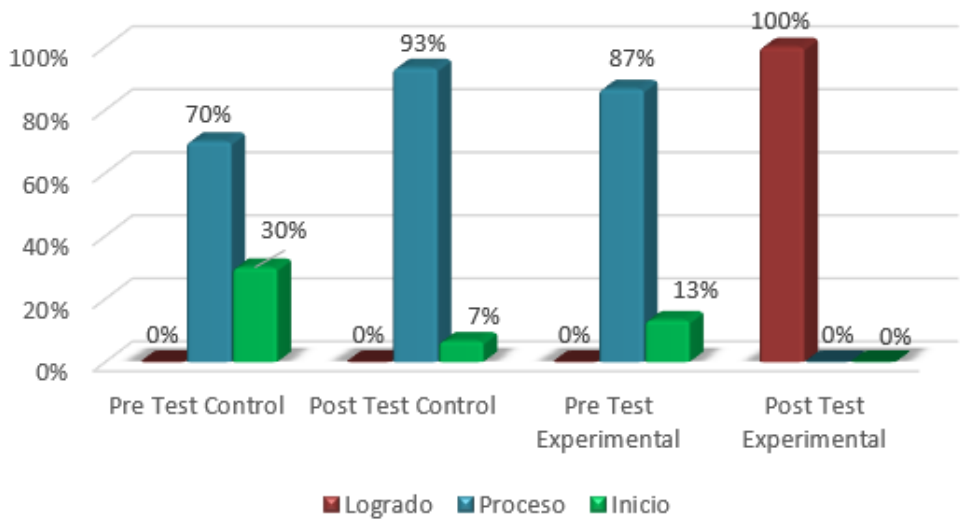

En cuanto a los resultados mostrados en la tabla y figura en referencia a la competencia procedimental, se muestra que antes de la aplicación de los videos tutoriales en el grupo control el 30\% llegó al nivel de inicio y el 70\% llegó al nivel de proceso, mientras que en el grupo experimental el 13\% se ubicó en el nivel de inicio y el $87 \%$ en el nivel de proceso.

Posteriormente se volvió a evaluar a ambos grupos obteniéndose que en el grupo control el 7\% se ubicó en el nivel de inicio y el $93 \%$ en proceso, mientras que en grupo experimental se tuvo que el $100 \%$ pudo llegar al nivel logrado.

Figura 4. Porcentajes de los niveles de la competencia actitudinal.

\section{COMPETENCIA ACTITUDINAL}

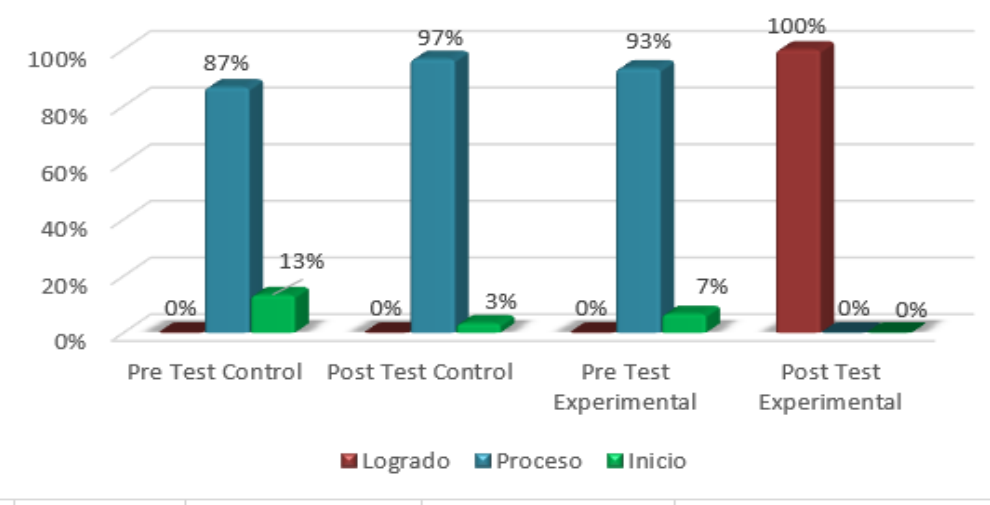

Finalmente, en cuanto a la competencia actitudinal los datos presentados en la tabla y figura con respecto a la evaluación realizada antes de la intervención en la que en el grupo control el 13\% de los estudiantes evaluados se ubicaron en el nivel inicio y el $87 \%$ en el nivel proceso; mientras que en grupo experimental el $7 \%$ se encuentra en el nivel de inicio y el $93 \%$ en el nivel de proceso.

Después de la intervención los resultados muestran que en el grupo control, el 7\% está en nivel inicio y el 93\% en el nivel de proceso, mientras que en el grupo experimental el 100\% llegó al nivel logrado. 


\section{CONCLUSIONES DE LA INVESTIGACIÓN}

El uso de video tutoriales mejora las competencias matemáticas de los estudiantes de Matemática de pregrado en las aulas virtuales de la USMP Virtual 2018, ya que el grupo experimental en el pretest tuvo un $23 \%$ de los estudiantes en el nivel de inicio, mientras en el pos-test el grupo logró el 100\% en el nivel logrado, lo cual se dio por la aplicación de los videos tutoriales.

El uso de los videos tutoriales mejora el desarrollo de la competencia conceptual de los estudiantes de Matemática de pregrado, ya que el grupo experimental en el pretest evidenció un $40 \%$ de los estudiantes se encontraban en el nivel de inicio, pero en el pos-test, del grupo experimental obtuvo el $100 \%$ de los estudiantes en el nivel logrado.

El uso de videos tutoriales mejora el desarrollo de la competencia procedimental de los estudiantes de Matemática de pregrado, porque se observó que el grupo experimental en el pretest obtuvo el $13 \%$ de los estudiantes que se encontraban en el nivel de inicio, pero en el pos-test del grupo experimental mostró que el $100 \%$ de los estudiantes se ubicaron en el nivel logrado.

El uso de videos tutoriales mejora el desarrollo de la competencia actitudinal de los estudiantes de Matemática de pregrado en las aulas virtuales de la USMP Virtual 2018, porque se pudo observar que el grupo experimental en el pretest logró un $7 \%$ de los estudiantes en el nivel de inicio, en cuanto al desarrollo de la competencia matemática, pero en el pos-test del grupo experimental obtuvo el $100 \%$ de los estudiantes en el nivel logrado. 


\section{REFERENCIAS}

Álvarez, J. Grau, S. Tortosa, M. (2016). Tecnologías de la Información y Comunicación Aplicadas a la educación Matemática (TICEM). Universidad de Alicante, Departamento Innovación y Formación Didáctica, España. ISBN: 978-84-608-4181-4.

Ausubel, D. (2002). Adquisición y retención del conocimiento. Una perspectiva cognitiva. España: Paidós.

Camposeco, T. (2012). La autoeficacia como variable en la motivación intrínseca y extrínseca en matemáticas a través de un criterio étnico. (Tesis de Doctor). De la Facultad de educación de Universidad Complutense de Madrid, España.

Bucio, M. (2014). Aprender a vivir. Maestría en Educación. Instituto "Antonio de Mendoza" y Preparatoria "Rector Hidalgo", Morelia.

Delors, M. (1996). Cuatro aprendizajes fundamentales. Comisión Internacional sobre educación para el siglo XX.

Gonzales, Y. (2013). El video tutorial como herramienta de apoyo pedagógico. Revista de la Universidad Autónoma del Estado de Hidalgo, México. Recuperado de https://www.uaeh.edu.mx/scige/boletin/prepa4/n1/e8.html

Gómez, R. (2012). Maestría en Enseñanza de las Ciencias Exactas y Naturales. Sensibilidad y aprendizaje de la matemática. Universidad Nacional de Colombia, Palmira, Colombia.

Huamán, M. (2012). La herramienta Cmap Tools mejora el aprendizaje colaborativo en el aula virtual USMP. Lima, Perú. Recuperado de http://hdl.handle.net/20.500.12579/3468 Repositorio Digital UNAM, México.

Huamán, M. (2015). Metodología para sesiones de clase on-line basada en un Modelo pedagógico e-learning propio de la USMP Virtual. Campus Virtual USMP. Universidad de San Martín de Porres, Lima, Perú.

Madueño, C. (2017). Aplicación del software Geogebra y su influencia en el nivel de conocimientos de. Maestría en Ciencias de la Educación. Universidad Nacional de Educación Enrique Guzmán y Valle, Lima.

Palomo, R., Ruiz, J. y Sánchez, J. (2008). Enseñanza con TIC en el siglo XXI. La escuela 2.0. Sevilla: MAD.

Siemens, G. (2004). Conectivismo: A Learning Theory for the Digital Age. Recuperado de http://www.elearnspace.org/Articles/connectivism.htm

Zúñiga, M. (2008). Estándares de desempeño de estudiantes en el aprendizaje con tecnologías digitales. Madrid: Fundación Omar Dazo. 